\title{
SIC/ALN PIEZOELECTRIC ENERGY HARVESTERS FOR PULSED PRESSURE SOURCES IN HARSH ENVIRONMENT APPLICATIONS
}

\author{
Yun-Ju Lai ${ }^{1 *}$, Wei-Chang Li ${ }^{l}$, Chih-Ming Lin ${ }^{l}$, Valery V. Felmetsger ${ }^{2}$, Debbie G. Senesky ${ }^{3}$, and Albert P. Pisano ${ }^{l}$ \\ ${ }^{1}$ Berkeley Sensor and Actuator Center (BSAC), University of California, Berkeley, California, USA \\ ${ }^{2}$ OEM Group Incorporated, Gilbert, Arizona, USA \\ ${ }^{3}$ Stanford University, Palo Alto, California, USA
}

\begin{abstract}
Piezoelectric micromachined circular diaphragm energy harvesters for pulsed pressure sources have been fabricated and characterized. These devices utilize silicon carbide $(\mathrm{SiC})$ as the structural material and aluminum nitride (AIN) as the active piezoelectric element to enable operation within harsh environments. In particular, the SiC/AIN energy harvesting device yields an output power density of $20 \mu \mathrm{W} / \mathrm{cm}^{2}$ and $\mathrm{rms}$ voltage of $0.2 \mathrm{~V}$ under pressure pulses of $1.09 \mathrm{psi}(\mathrm{rms})$ at $2 \mathrm{kHz}$. Fabricated $\mathrm{SiC} / \mathrm{AlN}$ harvester has been characterized at elevated temperatures as high as $250^{\circ} \mathrm{C}$. This work not only demonstrates the first harsh environment energy harvesters based on $\mathrm{SiC}$ and $\mathrm{AlN}$ for a pulsed pressure environment, but also addresses its potentials for integrating energy harvesting power source with SiC circuitry and enabling self-powered wireless sensors. This type of sensing system can be used for the real-time monitoring of harsh environment energy system such as automotive engines, gas turbines and geothermal wells.
\end{abstract}

\section{INTRODUCTION}

Condition-based monitoring of automotive engines, jet engines and gas turbines requires circuits and sensors, which have stable operation at elevated temperatures $\left(250^{\circ} \mathrm{C}\right.$ to $\left.600^{\circ} \mathrm{C}\right)$ and under harsh chemical environments (oxidizing, hydrocarbon, $\mathrm{CO}$ and $\mathrm{CO}_{2}$ ). This can aid in achieving efficient energy usage, reduced emissions and long lifetime for the combustion processes and critical components. In addition, harsh environment sensors also enable direct subsurface monitoring of geothermal and oil wells leading to enhanced energy recovery. However, at elevated temperatures, power supplies for electronic circuits and sensors are scarce. Current battery technology limits the working temperature range to below $150^{\circ} \mathrm{C}$ and cabled-power wiring dramatically increases the system complexity as well as the installation and maintenance cost.

To address this issue, energy harvesting [1][2] has emerged as promising power source, which can further lead to an autonomous integrated sensing module with reduced cost. Up to date, only a few energy harvesting devices for harsh environments have been reported, converting ambient vibration [3] and UV [4] energy into electrical energy. However, considering the availability of the ambient energies in the potential harsh environment systems, pulsed-pressure sources commonly existing in the combustion processes appears greatly attractive. In fact, the amount of the available energy from pressure pulsation in the target environment can be much larger than vibration sources and in addition, without requiring a UV-rich environment.

This work first demonstrates a piezoelectric SiC/AlN circular diaphragm energy harvester aiming to harvest the energy from pulsed-pressure sources in combustion systems. This micro-scale power source has the potential to achieve system integration with harsh environment circuit and sensor components [5][6]. To enable operation in harsh environments, the structural material selected for the energy harvesting devices consists of epitaxial cubic $\mathrm{SiC}$ (3C-SiC) and $\mathrm{AlN}$. SiC is used as the diaphragm structural material and AlN serves as the piezoelectric material for power

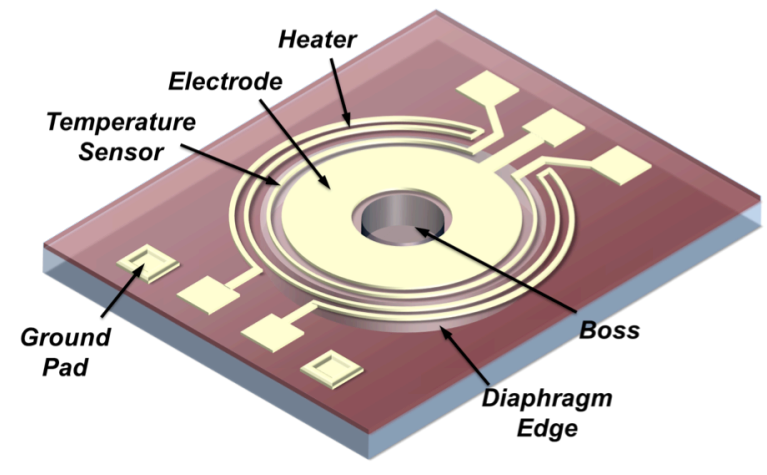

Figure 1: Schematic of a micromachined piezoelectric SiC/AlN bossed-diaphragm energy harvester with an on-chip thermal platform (resistive heaters and temperature sensors) for pulsed pressure sources.

conversion. Both appear favorable due to their excellent mechanical and electrical properties and resistance to chemical attack especially at elevated temperatures compared to their counterparts, e.g., silicon and PZT. By using this material combination, a micromachining process has been developed for fabricating the SiC/AlN composite diaphragm structure. The fabricated energy harvester with $4000-\mu \mathrm{m}$ diameter generates a power output as high as $2.5 \mu \mathrm{W}$, equivalent to a power density of approximately $20 \mu \mathrm{W} / \mathrm{cm}^{2}$, showing promise for providing power to most of the low-power sensor circuits. To investigate the functionality of the harvesters at elevated temperatures, the device equipped with an on-chip thermal platform (resistive heating and sensing elements) is measured and is shown to operate at temperatures above $200^{\circ} \mathrm{C}$. The experimental results not only show a SiC/AlN energy harvesting device with high output power density but also verifies its operation capabilities under high temperature environments. The harvester design, fabrication process, testing setup and measured results are presented as follows.

\section{SIC/ALN ENERGY HARVESTING DEVICE}

Piezoelectric AIN is chosen as the active material for power conversion rather than the commonly used PZT, which possesses large piezoelectric coefficients, for reasons that $\mathrm{AlN}$, which is non-ferroelectric and does not have a Curie point, retains its intrinsic piezoelectric properties even at high temperatures [7]. In fact, AlN has been demonstrated for a Lamb waver resonator to provide stable operation as high as $700^{\circ} \mathrm{C}$ [8]. Moreover, high quality AlN can be sputter-deposited at wafer-level with compatible processing along with SiC. The diaphragm structural material, $\mathrm{SiC}$, on the other hand, in addition to the mechanically robust nature under harsh environments, its superior electrical properties also enable SiC-based circuits and sensors for harsh environment electronics. Plus, $\mathrm{SiC}$ and $\mathrm{AlN}$ have well-matched mechanical and thermal properties, e.g., thermal expansion coefficients [9]. Platinum (Pt) and molybdenum (Mo) are the two 


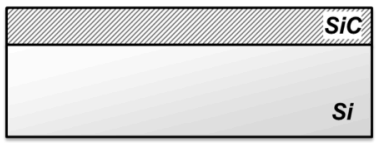

(b) Deposit Insulation $\mathrm{SiO}_{2}$

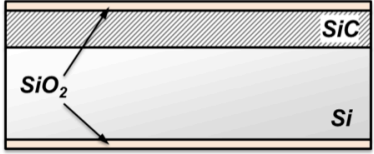

(c) Deposit Mo \& AIN

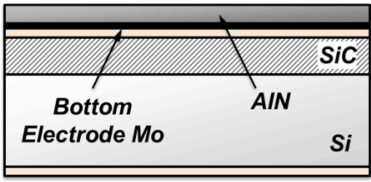

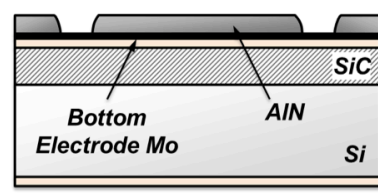

(e) Pattern Top Electrode Pt

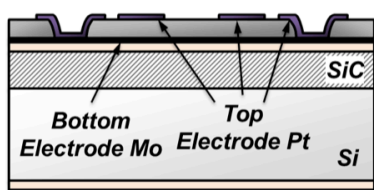

(f) Release Using DRIE

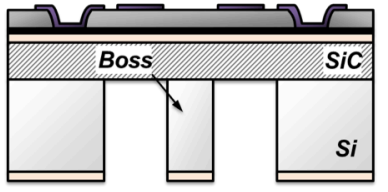

Figure 2: Fabrication process flow for the micromachined $\mathrm{SiC} / \mathrm{AlN}$ bossed-diaphragm energy harvesters.

metal materials utilized for the electrodes considering the high temperature survivability.

Figure 1 illustrates the design of a SiC/AlN energy harvesting device showing a circular bossed-diaphragm with patterned electrodes for electrical outputs as well as an on-chip thermal platform for high temperature measurements. The composite diaphragm is composed of a metal/AlN/metal sandwich layer on top of the structural $\mathrm{SiC}$ with a thin layer of $\mathrm{SiO}_{2}$ for electrical insulation in between. Transduction via $d_{31}$ piezoelectric coefficient is utilized for electrical power generation as the diaphragm deforms in response to the pressure pulses in the environments. The central boss structure adopted in this design can tune the mechanical resonant frequency of the composite diaphragm without modifying the diaphragm sizes. The electrode configurations (size and location) are determined to maximize the deliverable power generated by the piezoelectric AlN layer based on the in-plane strain distribution of the pressure-loaded diaphragm under large deformation. In addition to the electrode geometries, for maximizing the device output power, residual stress in the structural layers (AIN and $\mathrm{SiC}$ ) needs to be well controlled as it significantly reduces the diaphragm compliance [10]. Moreover, excellent crystallinity of the piezoelectric AlN thin film is required to achieve high electromechanical coupling and thus better power conversion.

\section{FABRICATION PROCESS}

Figure 2 depicts the bulk micro-machining process flow that is used to achieve the $\mathrm{SiC} / \mathrm{AlN}$ energy harvesting devices. A $\sim 3.2-\mu \mathrm{m}$-thick structural layer, $3 \mathrm{C}-\mathrm{SiC}$, is first deposited on a $\mathrm{Si}$ (100) substrate by epitaxial chemical vapor deposition with a residual stress below $100 \mathrm{MPa}$ by NOVASiC as shown in Fig. 2(a). A 500-nm oxide is then deposited via low pressure chemical vapor deposition as the insulation layer between $\mathrm{SiC}$ and bottom electrode (Fig. 2(b)). Next, well-textured bottom electrode Mo is sputtered on the insulation oxide with a thin layer of AIN as the seedlayer in order to achieve its high crystallinity for the following AlN growth. Highly $c$-axis-oriented AlN thin film $(\sim 1.0 \mu \mathrm{m}$, rocking curve FWHM $<1.5^{\circ}$, stress $\left.<200 \mathrm{MPa}\right)$ [11] is then

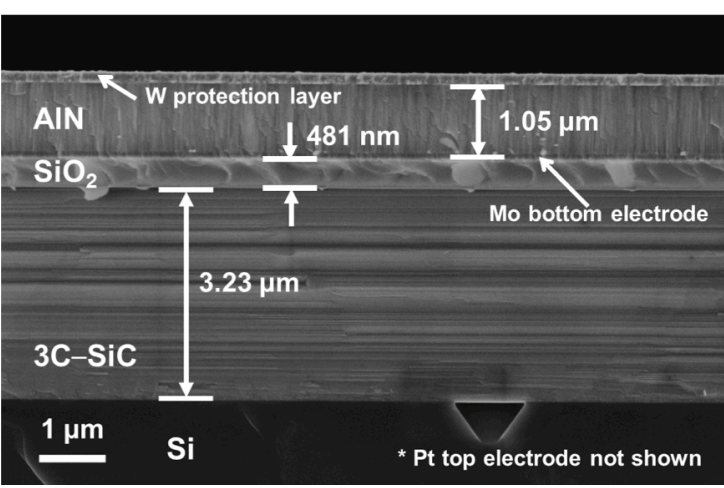

Figure 3: SEM of the cross-section of composite layer including piezoelectric AlN, bottom electrode Mo, insulation $\mathrm{SiO}_{2}$ and structural $3 \mathrm{C}-\mathrm{SiC}$.

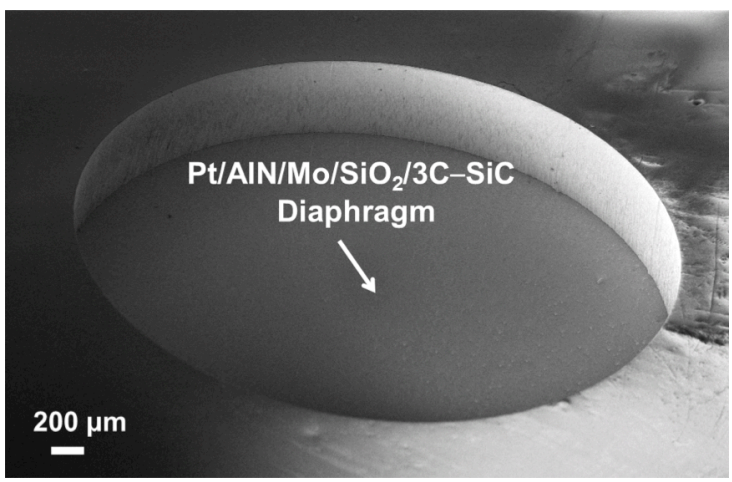

Figure 4: SEM of a released diaphragm structure (without boss) from the backside.

sputtered on blank Mo as shown in Fig. 2(c). A photolithography step followed by wet-etching AlN layer is used to access the bottom electrode (Fig. 2(d)). The top metal, Pt, is patterned on the AlN layer via a lift-off process to obtain the cross-section in Fig. 2(e). A backside photolithography step is then applied to create an etching mask for the diaphragm release step using $\mathrm{SF}_{6}$-based deep reactive ion etching (DRIE) and the final cross-section is shown in Fig. 2(f). The SEM of the cross-section of the composite layers is shown in Figure 3 and Figure 4 shows the SEM of a released energy harvester diaphragm (without boss) from the backside.

\section{MEASUREMENT SETUP}

\section{Pressure pulse generation}

In order to mimic the pulsed-pressure environment in the combustion chambers, an in-house pressure pulse generation unit is built. A commercial chopper (rotating blade with equally spacing slits) is utilized to modulate a static airflow and produce periodic pressure loads. The generated pressure pulse frequencies can be precisely controlled by both the chopper rotation speed and the number of blade slits. Figure 5 shows a time-domain waveform for $1-\mathrm{kHz}, 0.46-\mathrm{psi}$ pressure pulses measured by a commercial pressure sensor IC. The waveform shows the existence of higher frequency tones, which in fact present integer multiples of the prescribed frequency with smaller amplitude. Since the pressure amplitude generated is not constant due to the airflow fluctuations, the amplitude as well as the generated voltage from the harvester indicted in the following experimental results are presented as rms values for more than 100 cycles. 


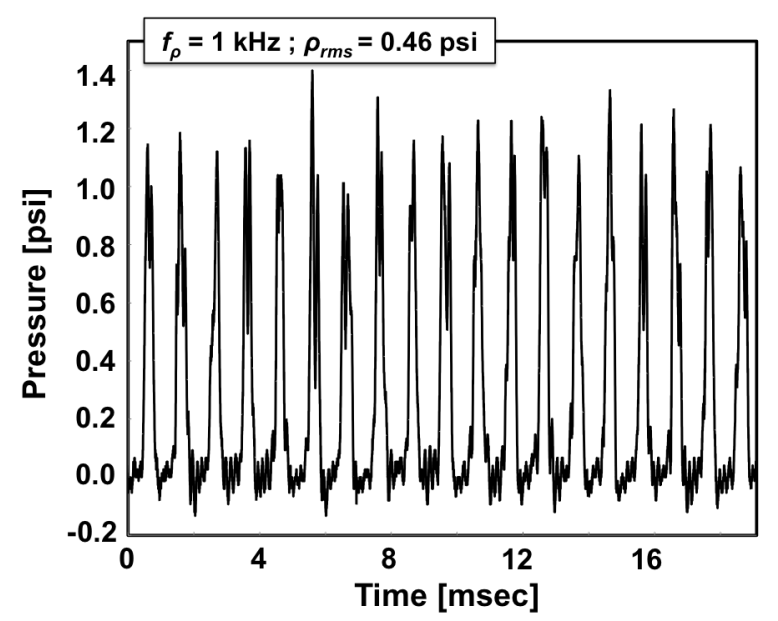

Figure 5: Measured waveform of 1-kHz, 0.46-psi (rms) pressure pulses from the pressure generation unit. .

\section{High temperature vibration testing}

In order to investigate the operation of the SiC/AlN energy harvesting device at elevated temperatures, the on-chip resistive heaters (fabricated along with the top metal electrode) are used to heat up the diaphragm via Joule heating to temperatures higher than $200^{\circ} \mathrm{C}$. The temperature of the diaphragm is then estimated by the variations of the temperature coefficients of resistance from another set of patterned top electrodes, forming the temperature sensor in the thermal platform. A shaker system is utilized in this case to provide vibration sources in order to measure the device responses with thermal elements since the cold pressure pulses generated from the setup discussed above prevent the heating of the device to high temperatures. It should be noted that the mechanical energy obtained from a vibrating shaker by the specific device demonstrated in this study is expected to be much less compared to that from pressure pulses and therefore, this measurement setup is only used to characterize the device operation under elevated temperatures and will not be able to reveal the power generation capabilities of the energy harvesting devices.

\section{EXPERIMENTAL RESULTS}

The devices are first characterized in the pulsed pressure environment as discussed in the previous section at room temperature. By connecting the harvester to a pure resistive load, time-varying voltages across the load are measured from an oscilloscope under various pressure amplitudes and load resistances. Figure 6 and 7 present the generated rms voltage and average power, respectively versus the load resistances from a device of $3500 \mu \mathrm{m}$ in diameter under $1-\mathrm{kHz}$ pressure pulses with varying amplitudes. With the given geometries of the diaphragm without central boss, the device is estimated to operate far from its resonance with $1-\mathrm{kHz}$ pressure pulses. The optimal load resistance, depending on the size of the active area as well as the source and resonance frequency of the device, is required in order to achieve maximum delivered power. A maximum power of $26 \mathrm{nW}$ is shown to be delivered to a $90-\mathrm{k} \Omega$ resistive load under a $0.46-\mathrm{psi}$ pressure with rms output voltage of $0.049 \mathrm{~V}$. Note that the small power output presented here is due to the low level of pressure amplitude applied as well as the far-from-resonance operation.

Lowering the device resonant frequency by introducing the central boss to the diaphragm with size properly determined, the harvested power can be greatly improved. Figure 8 shows the

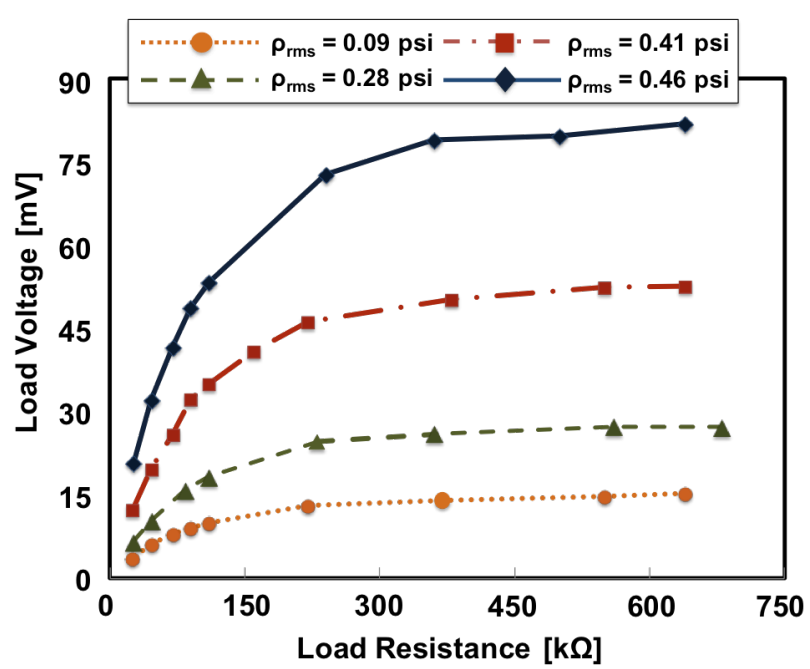

Figure 6: Measured generated voltages versus load resistance under varying pressure amplitude at a fixed $1-\mathrm{kHz}$ frequency. The device is $3500 \mu \mathrm{m}$ in diameter without boss and electrode size is $3100 \mu \mathrm{m}$.

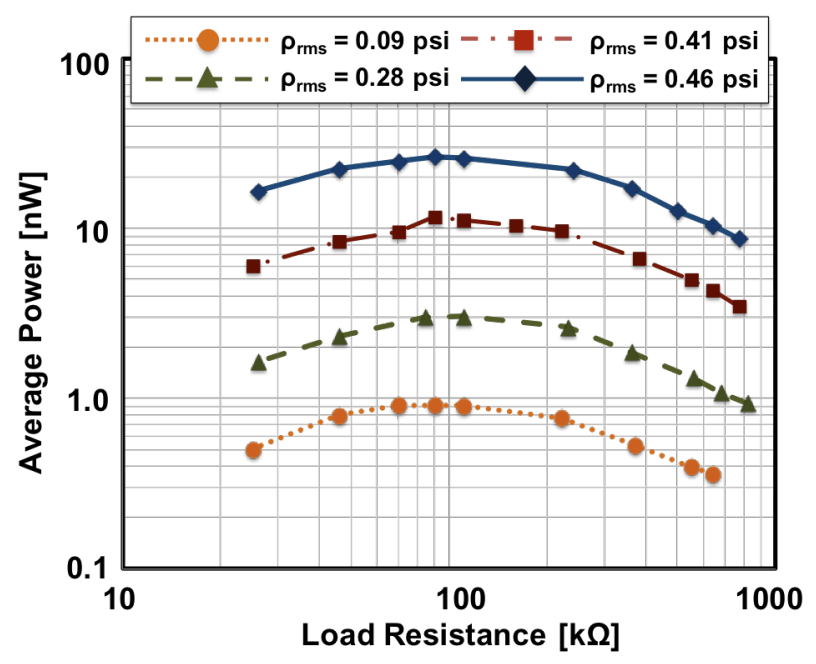

Figure 7: Measured average power versus load resistance under varying pressure amplitude at a fixed $1-\mathrm{kHz}$ frequency. The device is $3500 \mu \mathrm{m}$ in diameter without boss and electrode size is $3100 \mu \mathrm{m}$.

response of a device with a $4000-\mu \mathrm{m}$ diaphragm and a central boss of $1500 \mu \mathrm{m}$ producing rms voltage of $0.2 \mathrm{~V}$ and an average power of $2.5 \mu \mathrm{W}$ with a $26-\mathrm{k} \Omega$ load under $2-\mathrm{kHz}$ pressure pulses with pressure amplitude of $1.09 \mathrm{psi}$ (rms), corresponding to a power density of $\sim 20 \mu \mathrm{W} / \mathrm{cm}^{2}$. Though the prescribed frequency is still lower than the resonance frequency (estimated to be $10 \mathrm{kHz}$ ), the device is excited at resonance by higher frequency tones of the prescribed frequency with reduced amplitude. Based on these results, it can be expected that by matching the device resonant frequency with the pressure source frequency, increased power can be generated with design improvements.

\section{High temperature measurement}

To characterize the energy harvesters' response at elevated temperatures, the devices with central boss, which can be easier to actuate with acceleration from a shaker system, are measured under vibration at temperatures up to $250^{\circ} \mathrm{C}$, achieved by the 


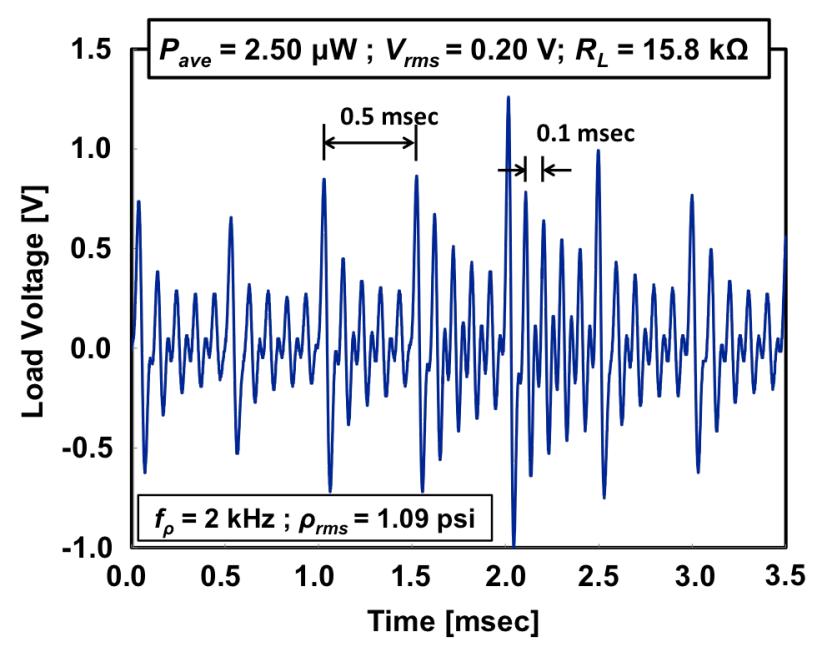

Figure 8: Measured output voltage waveform on a 15.8- $k \Omega$ load under 2-kHz, 1.09-psi (rms) pressure pulses. The device is $4000 \mu \mathrm{m}$ in diameter with a 1500- $\mu \mathrm{m}$ central boss and 1125- $\mu$ m-wide electrode.

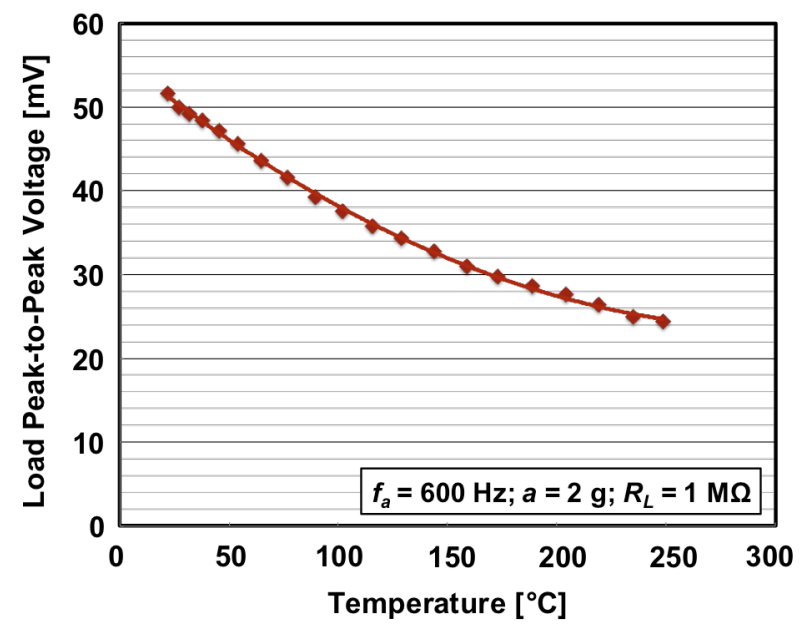

Figure 9: Measured peak-to-peak voltage on a 1-MS load resistor versus temperatures. The device is $3000 \mu \mathrm{m}$ in diameter with a 500- $\mu \mathrm{m}$ central boss and 990- $\mu \mathrm{m}$-wide electrode.

on-chip heater as previously described in the measurement setup section. Figure 9 shows the generated peak-to-peak voltage versus temperatures from $22^{\circ} \mathrm{C}$ to $250^{\circ} \mathrm{C}$ on a $1-\mathrm{M} \Omega$ resistive load for the device under a $2-\mathrm{g}$ vibration. The presented data is obtained with the harvester experiencing a number of thermal cycling until reaching stable operation. It can be noticed in Figure 9 that the output voltages degrade as temperature increases and possible reasons leading to the degradation are the induced thermal stress in the diaphragm as well as the increased thermal resistances from the metal. More importantly, the device is able to generate power at temperatures above $200^{\circ} \mathrm{C}$ and confirms that the AlN remains piezoelectric under these conditions.

\section{CONCLUSIONS}

An output power density of approximately $20 \mu \mathrm{W} / \mathrm{cm}^{2}$ has been obtained with the micromachined piezoelectric $\mathrm{SiC} / \mathrm{AlN}$ bossed-diaphragm energy harvesters under $2-\mathrm{kHz}$ pressure pulses of $1.09 \mathrm{psi}(\mathrm{rms})$. Although increased electrical power output can be expected by matching the pressure pulse frequency to the diaphragm mechanical resonant frequency, the power density level is already sufficient for the need in most of low-power sensor circuits. Under elevated temperatures up to $250^{\circ} \mathrm{C}$, measurements confirm that the energy harvesting devices are still able to generate electrical power with reduced output levels compared to that of room temperature. This shows that the fabricated SiC/AlN energy harvesting device for pulsed-pressure sources is a promising power source for harsh environment circuits and sensors. Obviously, further investigations are needed to understand the causes of power degradation at elevated temperatures and to ensure functionality in more stringent environments.

\section{ACKNOWLEDGEMENTS}

The authors would like to acknowledge the Marvell Nanofabrication Laboratory staff in the University of California at Berkeley for their processing assistance and the Transducer Research Foundation for the generous travel support.

\section{REFERENCES}

[1] S.P. Beeby, M.J. Tudor and N.M. White, "Energy Harvesting Vibration Sources for Microsystems Applications," Meas, Sci, Technol. 17, pp. 175 - 195, 2006.

[2] P.D. Mitcheson, et al., "Energy Harvesting from Human and Machine Motion for Wireless Electronic Devices," Proc. of the IEEE vol. 96, no. 9, pp. 1457 - 1486, 2008.

[3] S.B. Barker, K.V. Vassilevski, N.G. Wright and A.B. Horsfall, "High Temperature Vibration Energy Harvester System," Proc. IEEE Sensors Conf., pp. 300 - 303,2010.

[4] S.B. Barker, et al., "Silicon Carbide based Energy Harvesting Module for Hostile Environments," Materials Science Forum vol. 645-648, pp. 1093 - 1096, 2010.

[5] A.C. Patil, et al., "6H-SiC JFETs for $450^{\circ} \mathrm{C}$ Differential Sensing Applications," J. Microelectromechanical Systems, vol. 18 , no. 4, pp. 950 - 961, 2009.

[6] R.G. Azevedo, D.G. Senesky, A.V. Jog, B. Jamshidi, D.R. Myers, L. Chen, X.-A. Fu, M. Mehregany, M.B.J. Wijesundara, and A.P. Pisano, "A SiC MEMS Resonant Strain Sensor for Harsh Environment Applications," IEEE Sensors J., vol. 7, No. 4, pp. 568 - 576, 2007.

[7] R. Farrell, et al., "High Temperature Annealing Studies on the Piezoelectric Properties of Thin Aluminum Nitride Films," Proc. of Mater. Res. Soc. Symp., vol. 1052, no. 1052-DD06-18, 2008.

[8] C.-M. Lin, T.-T. Yen, V.V. Felmetsger, M.A. Hopcroft, J.H. Kuypers, and A.P. Pisano, "Thermally Compensated Aluminum Nitride Lamb Wave Resonators for High Temperature Applications," Appl. Phys. Lett., vol. 97, 083501, 2010.

[9] V.V. Luchinin, A.V. Korlyakov, and A.A. Vasilev, "Silicon Carbide-Aluminium Nitride: a New High Stability Composition for MEMS," Proc. SPIE, vol. 3680, pp. 783 791, 1999.

[10] J.H. Jerman, "The Fabrication and Use of Micromachined Corrugated Silicon Diaphragms," Sensors and Actuators, A21-A23, pp. 988 - 992, 1990.

[11] V.V. Felmetsger, P.N. Laptev, and S.M. Tanner, "Innovative Technique for Tailoring Intrinsic Stress in Reactively Sputtered Piezoelectric Aluminum Nitride Films," J. Vac. Sci. Technol. A, vol. 27, no. 3, pp. 417 - 422, 2009.

\section{CONTACT}

*Y.-J. Lai, Tel: +1-510-643-9752; matildal@eecs.berkeley.edu 